\title{
Avaliação da qualidade de vida de acadêmicos de graduação em Enfermagem do primeiro e quarto anos: influência das variáveis sociodemográficas
}

\author{
Evaluation of quality of life of undergraduate nursing students from first and fourth \\ years: the influence of sociodemographic variables
}

\author{
Rosane Bueno Eurich ${ }^{1}$, Ana Cláudia G. C. Kluthcovsky ${ }^{2}$ \\ ${ }^{1}$ Enfermeira, Unidade Mista de Humaitá, Humaitá, AM. ${ }^{2}$ Mestrado. Médica, Professora assistente, Universidade Estadual do Centro-Oeste, \\ Guarapuava, PR.
}

\section{Resumo}

Introdução: O objetivo deste artigo foi avaliar a qualidade de vida de acadêmicos de graduação em Enfermagem de uma universidade pública do Estado do Paraná, correlacionar com variáveis sociodemográficas, e comparar os acadêmicos do primeiro e quarto anos do curso.

Método: Estudo descritivo, transversal e com abordagem quantitativa. Participaram 34 acadêmicos do primeiro ano (89,5\%) e 33 acadêmicos do quarto ano (100\%), que responderam ao questionário WHOQOL-bref da Organização Mundial da Saúde, além de informações complementares.

Resultados: Os acadêmicos pesquisados eram predominantemente mulheres, jovens, solteiras, com renda per capita média familiar mensal de mais de um salário mínimo, procedentes de outras cidades e residiam com a família ou em república/pensionato. Considerando todos os acadêmicos, o maior escore médio de qualidade de vida foi para o domínio físico $(72,7+13,1)$, e o menor para o domínio meio ambiente $(60,7+12,7)$. Na correlação com variáveis sociodemográficas, apenas o sexo apresentou correlação com a qualidade de vida, com maiores escores médios para os acadêmicos do sexo masculino nos domínios físico $(79,9+9,8)$ e psicológico $(77,6+6,3)$. Na análise comparativa entre os acadêmicos do primeiro e quarto anos, apesar das diferenças significativas para idade e renda, com o primeiro ano apresentando alunos mais jovens e com menor renda média familiar mensal em relação ao quarto ano, não houve diferença significativa nos domínios da qualidade de vida.

Conclusão: Os resultados deste estudo sugerem que os acadêmicos de Enfermagem merecem atenção quanto à qualidade de vida, principalmente as mulheres nos aspectos físico e psicológico.

Descritores: Qualidade de vida, estudantes de enfermagem, Organização Mundial da Saúde, estudos transversais.

\begin{abstract}
Introduction: The aim of this study was to evaluate the quality of life of undergraduate students from a nursing course in a public university in the State of Paraná, Brazil, correlating with sociodemographic variables, and to compare undergraduate students from first and fourth years.

Method: This is a descriptive and cross-sectional study with a quantitative approach. Thirty-four students (89.5\%) from the first year and $33(100 \%)$ from the fourth year participated. They answered the WHOQOL-bref, a questionnaire designed by the World Health Organization, in addition to some additional information.
\end{abstract}

\footnotetext{
Correspondência:

Ana C. G. C. Kluthcovsky, Departamento de Enfermagem, Rua Simeão Varela de Sá, 3, Bairro dos Estados, CEP 85040-080, Guarapuava, PR. Email: anafabio@brturbo.com.br 
Results: The students were predominantly female, young and single, with per capita family income up to one minimum wage, coming from other cities and living with the family or with housemates. Considering the entire sample, the highest quality of life mean score was for the physical domain $(72.7+13.1)$, and the lowest for the environment domain $(60.7+12.7)$. Correlation with sociodemographic variables showed that only gender had significant correlation with quality of life. The highest mean scores were for male students in the physical $(79.9+9.8)$ and psychological $(77.6+6.3)$ domains. There was significant difference between age and income in the comparison between students from first and fourth years, the profile of first-year students being younger and with low income than students from the fourth year. In spite of this, no significant difference in quality of life domains was found.

Conclusion: The results of this study suggest that nursing students need attention in relation to the quality of life, especially women in the physical and psychological aspects.

Keywords: Quality of life, nursing students, World Health Organization, cross-sectional studies.

\section{Introdução}

$\mathrm{Na}$ universidade, o indivíduo encontra um ambiente favorável para desenvolver e aprimorar valores relacionados à sua futura vida profissional $\mathrm{e}$ pessoal. Pela busca do conhecimento científico, deve encontrar condições para o desenvolvimento da consciência crítica e do seu papel enquanto cidadão, essenciais para uma formação profissional de compromisso com a sociedade ${ }^{1}$.

Segundo Saupe et al. ${ }^{2}$, entende-se acadêmico de Enfermagem como "um ser humano que fez uma opção de vida de cuidar e ajudar outros seres humanos: a nascer e viver de forma saudável, a superar agravos à sua saúde, a conviver com limitações e encontrar um significado nessa experiência, e a morrerem com dignidade. E que, no processo de preparar-se para realizar as várias ações que integram esse trabalho, com competência técnica, dialógica e política, enfrenta situações de sofrimento que podem contribuir tanto para seu processo de humanização, quanto para a banalização das mesmas".

$\mathrm{Na}$ formação do enfermeiro há maior ênfase na dimensão técnica, o que possibilita pouco crescimento interno do profissional, apesar da busca da integralidade do ser humano ${ }^{3}$. $\mathrm{O}$ apoio ao estudante está geralmente voltado apenas aos aspectos pedagógicos e à assistência emergencial e curativa, com pouca preocupação relacionada aos aspectos da saúde mental, o que não os ajuda a desenvolver a consciência e capacidade de reflexão, que seria útil para gerar oportunidades de aprendizado ou resolver situações difíceis de seu cotidiano e na futura profissão ${ }^{4}$.

$\mathrm{O}$ acadêmico de Enfermagem se encontra em uma fase da vida com possibilidades de mudanças e de novas expectativas, o que pode causar profundos reflexos na sua qualidade de vida atual e futura ${ }^{5}$. Assim, a discussão sobre o processo de formação dos cuidadores profissionais nas instituições formadoras é de extrema importância, principalmente considerandose as Diretrizes para a Educação em Enfermagem no Brasil. Nesse contexto, entende-se a necessidade de projetos que envolvam o sistema formador como um espaço articulador sobre a qualidade de vida do futuro profissional ${ }^{6}$.

O termo qualidade de vida é de difícil conceituação, e ainda não se chegou a um consenso sobre ele, apesar dos debates nas últimas décadas ${ }^{7-10}$. Mesmo com as dificuldades conceituais, é crescente o interesse e a ênfase de muitos autores sobre a necessidade e a importância da avaliação da qualidade de vida, inclusive na área da saúde ${ }^{9-10}$.

Ao longo do tempo, o conceito foi ampliado, paulatinamente englobando o desenvolvimento socioeconômico e humano, ou aspecto objetivo, e a percepção individual, ou aspecto subjetivo ${ }^{9}$. Além disso, a percepção da qualidade de vida é variável de pessoa para pessoa, e é dinâmica em cada indivíduo ${ }^{11}$.

Sobre as diversas abordagens da qualidade de vida, Holmes \& Dickerson ${ }^{12}$ relatam que os fatores que contribuem para a qualidade de vida, como um todo, são: satisfação pessoal, auto-estima, capacidade de desempenho, comparação com os outros, conhecimentos e experiências prévias, condições econômicas, estado geral de saúde e estado emocional.

Com relação às definições de qualidade de vida, observa-se um grande número na literatura, e, para Meeberg $^{8}$, algumas focalizam os aspectos subjetivos, outras os objetivos, e outras utilizam ambos, de maneira unidimensional ou multidimensional. Alguns autores definem qualidade de vida em termos de satisfação com a vida ou satisfação das necessidades.

Segundo a Organização Mundial da Saúde ${ }^{13}$ (OMS), qualidade de vida é definida como: "a percepção do indivíduo de sua posição na vida no contexto da cultura e sistemas de valores, nos quais ele vive e em relação a seus objetivos, expectativas, padrões e preocupações".

Neste estudo, ressalta-se a importância de avaliar a qualidade de vida em acadêmicos de Enfermagem, pelas características próprias dessa fase da vida.

Durante sua formação na universidade, os estudantes passam por processos de adaptação que podem gerar situações de crises, com o surgimento de depressões, alcoolismo, evasão escolar, dificuldades na aprendizagem, nos relacionamentos pessoais, e isolamento ${ }^{4}$. 
Além do seu cotidiano como acadêmicos, outros aspectos de sua vida também podem influenciar sua qualidade de vida e futuro profissional. Saupe et al. ${ }^{2}$ relatam algumas preocupações dos docentes em relação aos acadêmicos, como aprendizagem, interesse, motivação, presença de sofrimento do viver genérico relacionado a problemas financeiros, familiares, de saúde, e do cotidiano específico da futura profissão, carregado de intimidade com a dor e a morte da clientela usuária dos serviços de saúde, além do sistema predominante no ensino de graduação, "cujo discurso humanístico nem sempre começa na sala de aula".

Outro fator que reforça a relevância deste estudo é a escassez de publicações sobre o tema. Assim, este estudo teve como objetivos avaliar a qualidade de vida de acadêmicos de graduação em Enfermagem de uma universidade pública do Estado do Paraná, correlacionar com variáveis sociodemográficas, e proceder às comparações entre os acadêmicos do primeiro e quarto anos do curso.

\section{Método}

Este é um estudo descritivo, transversal e de abordagem quantitativa, do qual participaram acadêmicos em Enfermagem de uma universidade pública do Estado do Paraná. Trata-se de um curso realizado em período integral, com duração de quatro anos.

Apesar das limitações do estudo transversal, como impossibilidade de estabelecer causa e efeito, é um método muito usado nos estudos em que se pretende examinar a relação entre eventos. Também é um método rápido, simples, de baixo custo, e objetivo na coleta de dados ${ }^{14}$.

A universidade onde foi realizado o estudo localiza-se em um município de médio porte do Estado do Paraná, na Região Sul do Brasil. O município é pólo regional, sendo referência na região para a área da saúde e para a área de ensino, pois oferece diversos cursos universitários, em instituições tanto públicas quanto privadas, atraindo muitos estudantes de outras cidades, inclusive de outros Estados do país.

Foi solicitada autorização da coordenação do curso de Enfermagem para a realização dessa investigação, e o estudo foi analisado e aprovado pelo Comitê de Ética em Pesquisa da instituição.

Foi realizado um estudo piloto com oito acadêmicos de Enfermagem, todos da mesma universidade onde foi realizada a pesquisa, que visou testar a aceitação da pesquisa e o entendimento do questionário por parte dos participantes. Como não houve dificuldades durante a coleta, os dados do estudo piloto foram incluídos nos resultados finais.

Todos os participantes do estudo ou seus responsáveis legais, inclusive os do estudo piloto, assinaram o Termo de Consentimento Livre e Esclarecido, nos termos da Resolução 196/96 do Conselho Nacional de Ética em Pesquisa ${ }^{15}$.

A coleta de dados foi realizada durante os intervalos de aula, em um tempo destinado para essa investigação, durante os meses de abril, maio e junho de 2007.

O grupo de estudo foi constituído por acadêmicos matriculados no primeiro e quarto anos do curso de graduação em Enfermagem que aceitaram responder ao questionário para coleta de dados. Assim, dos 38 acadêmicos matriculados no primeiro ano do curso, 34 $(89,5 \%)$ participaram do estudo e quatro $(10,5 \%)$ se recusaram. Dos 33 acadêmicos matriculados no quarto ano, todos $(100 \%)$ participaram do estudo.

Optou-se pelo estudo do primeiro e quarto anos em função das diferenças específicas desses dois períodos de vida do acadêmico quanto às respectivas grades curriculares. No primeiro ano, os acadêmicos desenvolvem as disciplinas obrigatórias de formação básica. Os acadêmicos do quarto ano desenvolvem as disciplinas obrigatórias de formação profissional, incluindo estágios supervisionados em serviços de saúde, com contato direto com o paciente.

As informações do questionário foram preenchidas pelos próprios sujeitos da pesquisa, em um único encontro, e os questionários foram distribuídos e recolhidos pelas autoras.

$\mathrm{O}$ instrumento para coleta de dados utilizado foi o questionário genérico sobre qualidade de vida da OMS, o WHOQOL-bref, que consiste na versão abreviada do WHOQOL-100, ambos desenvolvidos pelo Grupo de Qualidade de Vida da $\mathrm{OMS}^{16}$. O WHOQOL-100 resultou de um projeto colaborativo multicêntrico com objetivo de construir um instrumento que avaliasse a qualidade de vida em uma perspectiva internacional ${ }^{17}$.

A versão em português do WHOQOL-bref foi desenvolvida no Centro WHOQOL para o Brasi1 ${ }^{16}$. Ela contém 26 questões: duas questões gerais, que não entram no cálculo dos escores dos domínios, e 24 distribuídas em quatro domínios: físico, psicológico, relações sociais e meio ambiente.

Os escores finais de cada domínio consideram as respostas de cada questão que compõe o domínio, resultando em escores finais numa escala de 4 a 20 , que podem ser transformados em 0 a 100, medidos em direção positiva. Escores mais altos indicam melhor avaliação da qualidade de vida.

A escolha do WHOQOL-bref ocorreu por ter sido desenvolvido e validado mostrando características psicométricas satisfatórias ${ }^{16}$, muito importantes na 
escolha de um instrumento para avaliação da qualidade de vida ${ }^{9,18,19}$. Além disso, pode ser utilizado tanto para populações saudáveis como para populações acometidas por agravos e doenças crônicas ${ }^{20}$.

Também foram coletadas informações complementares sobre o ano do curso em que o acadêmico estava matriculado, sexo, idade, estado civil, renda familiar e números de pessoas que viviam com essa renda, procedência e convívio domiciliar.

As variáveis foram analisadas por meio de freqüências absoluta e relativa, e medidas estatísticas descritivas. Após checagem de normalidade das variáveis pelo teste de Kolmogorov-Smirnov, foi utilizado o teste $t$ de Student para comparar médias. O teste Exato de Fisher foi utilizado para comparar proporções. O nível de significância estatístico adotado foi de 5\%.

\section{Resultados e discussão}

Os resultados referem-se à avaliação da qualidade de vida de 67 acadêmicos de Enfermagem pesquisados, sendo 34 acadêmicos do primeiro ano e 33 do quarto ano do curso.

O tempo médio para preenchimento dos questionários foi de 7 minutos ( $\mathrm{DP} \pm 3,1$ ).

O WHOQOL-bref, para utilização neste estudo, foi submetido à avaliação de confiabilidade, através da consistência interna, pelo coeficiente Alfa de Cronbach, com valores de 0,90 (para os 26 itens), 0,89 (para os domínios), 0,77 (domínio físico), 0,68 (domínio psicológico) e 0,77 (domínio meio ambiente). O domínio relações sociais apresentou coeficiente alfa de Cronbach de 0,51, apesar disso, optou-se por manter esse domínio nos cálculos, por se tratar de um domínio mais instável psicometricamente, como já demonstrado em teste de campo ${ }^{16}$.

A Tabela 1 apresenta a distribuição do total dos acadêmicos pesquisados, segundo variáveis sociodemográficas.

Quanto ao sexo dos pesquisados, as mulheres eram maioria $(80,6 \%)$. Estudos demonstram a predominância de mulheres nos cursos de Enfermagem, com percentuais variando de $89 \%$ a $97,8 \%{ }^{21-27}$. Esse perfil também foi confirmado em pesquisa sobre os estudantes que realizaram o Exame Nacional de Desempenho de Estudantes de 2004, que reforçou duas tendências já constatadas na literatura quanto às relações construídas historicamente entre a mulher e o cuidar, e socialmente entre a mulher e a opção pelos cursos de Enfermagem ${ }^{28}$.

A idade média dos acadêmicos foi 21,2 anos (DP $\pm 4,3$ ), com idade mínima de 17 e máxima de 44 anos. Pelos dados da Tabela 1, observa-se que a maioria dos acadêmicos $(53,7 \%)$ apresentava até 20 anos de idade.
Média de idade semelhante (20 anos) foi encontrada em pesquisa sobre depressão em 99 discentes de Enfermagem do primeiro ao quarto ano, da Faculdade de Medicina de Botucatu-Unesp, São Paulo ${ }^{26}$.

Em alunos de graduação em Enfermagem, pesquisados a respeito do conhecimento sobre terapias alternativas/complementares, em escola pública e privada, a média de idade foi de $24,3 \operatorname{anos}^{23}$.

Estudo do perfil sociodemográfico de alunos ingressantes no Curso de Enfermagem da Escola de Enfermagem de Ribeirão Preto-USP, de 1999 a 2003, demonstrou limites de idade de 16 a 45 anos, e faixa etária de 17 a 21 anos representando $92,04 \%$ do total. É possível que o ingresso de estudantes jovens, em ambos os estudos, se deva ao fato de serem cursos em tempo integral, o que dificulta ou impossibilita conciliar trabalho e estudo ${ }^{21}$.

Em avaliação de qualidade de vida de 264 acadêmicos de Enfermagem em um município do Estado de São Paulo, a idade variou de 17 a 40 anos, com predomínio também da faixa jovem, de 17 a 20 anos, em $65,5 \%$ da amostra $^{22}$.

Estudo recente com 224 acadêmicos do primeiro, segundo e terceiro anos do curso de graduação em Enfermagem, demonstrou que a idade variou de 17 a 44 anos, contudo, houve predominância da faixa etária de 21 a 25 anos $(60,3 \%)^{24}$.

Tabela 1 - Distribuição do total dos acadêmicos de Enfermagem estudados, segundo variáveis sociodemográficas (Paraná, 2007, n =67)

\begin{tabular}{|c|c|}
\hline Variáveis & n $(\%)$ \\
\hline \multicolumn{2}{|l|}{ Sexo } \\
\hline Feminino & $54(80,6)$ \\
\hline Masculino & $13(19,4)$ \\
\hline \multicolumn{2}{|l|}{ Idade } \\
\hline Até 20 anos & $36(53,7)$ \\
\hline$>20$ anos & $31(46,3)$ \\
\hline \multicolumn{2}{|l|}{ Estado civil } \\
\hline Solteiro(a) & $61(91,0)$ \\
\hline Casado(a) & $6(9,0)$ \\
\hline \multicolumn{2}{|c|}{ Renda per capita média familiar mensal* } \\
\hline Até $1 \mathrm{SM}$ & $15(23,4)$ \\
\hline Mais de $1 \mathrm{SM}$ & $49(76,6)$ \\
\hline \multicolumn{2}{|l|}{ Procedência } \\
\hline Cidade local & $26(38,8)$ \\
\hline Outra cidade & $41(61,2)$ \\
\hline \multicolumn{2}{|l|}{ Com quem reside } \\
\hline Sozinho(a) & $9(13,4)$ \\
\hline Familiares/outro & $58(86,6)$ \\
\hline
\end{tabular}

* Três dos respondentes não informaram. 
Quanto ao estado civil, os solteiros(as) corresponderam a $91,0 \%$. Uma maioria de acadêmicos solteiros também foi encontrada em estudo realizado entre alunos ingressantes no curso de graduação em Enfermagem $(96,8 \%)^{21}$, entre todos os alunos do curso $(88,6 \%)^{22}$, alunos do primeiro, segundo e quarto anos $(95,6 \%)^{24}$, e entre os alunos do último período do curso $(91,3 \%)^{25}$.

A grande maioria dos acadêmicos $(76,6 \%)$ possuía uma renda média familiar de mais de um salário mínimo por mês. Considerando a renda como números de salários mínimos recebidos pela família de cada acadêmico, a maior parte (45,5\%) tinha renda de 6 a 10 salários mínimos por mês.

Em estudo de avaliação de qualidade de vida já mencionado, realizado com alunos do curso de graduação em Enfermagem do Estabelecimento de Ensino Superior Integrado da Fundação Educacional de Fernandópolis, Estado de São Paulo, houve predominância de renda entre 6 a 10 salários mínimos, $43,9 \%$ dos respondentes, seguidos de $28,4 \%$ com renda de 11 ou mais salários mínimos. A maior parte dos estudantes possuía casa própria e o uso do automóvel como principal meio de transporte da família, o que evidenciou um bom poder aquisitivo das famílias dos estudantes $^{22}$.

Também entre 99 discentes de Enfermagem do primeiro ao quarto ano da Faculdade de Medicina de Botucatu-Unesp, São Paulo, a renda mensal familiar foi semelhante, de 5 a 10 salários mínimos ${ }^{26}$.

A maioria dos acadêmicos $(61,2 \%)$ procedia de outras cidades e, quanto ao convívio familiar, $86,6 \%$ residiam com familiares ou em pensionato/república, e apenas $13,4 \%$ residiam sozinhos. Em estudo já mencionado, a maioria dos estudantes $(95,96 \%)$ também não procedia da cidade onde estudava, e as formas de habitação mais freqüentes eram as repúblicas $(65,7 \%)^{26}$. De modo diferente, entre 33 alunos ingressantes em Enfermagem de uma universidade particular da cidade de São Paulo, mais da metade dos alunos $(52,0 \%)$ morava na zona sul, local da sede da faculdade, indicando sua forte inserção na comunidade a que pertence, complementada pela rede de transporte da localidade ${ }^{27}$.

Quanto aos escores médios atribuídos aos domínios da qualidade de vida (Tabela 2) o maior escore médio foi para o domínio físico 72,7 (DP $\pm 13,1$ ), e o menor escore médio para o domínio meio ambiente (DP $\pm 12,7)$.

Tabela 2 - Escores médios, mínimos e máximos dos domínios da qualidade de vida do WHOQOL-bref para o total de acadêmicos de Enfermagem estudados (Paraná, 2007, n =67)

\begin{tabular}{lccc}
\hline Domínios & Escore médio (DP) & Mínimo & Máximo \\
\hline Físico & $72,7(13,1)$ & 39,3 & 100,0 \\
Psicológico & $69,3(12,3)$ & 41,7 & 91,7 \\
Relações sociais & $71,3(15,7)$ & 41,7 & 100,0 \\
Meio ambiente & $60,7(12,7)$ & 21,9 & 87,5 \\
\hline
\end{tabular}

$\mathrm{DP}=$ desvio padrão.

Em estudo realizado por Saupe et al. ${ }^{2}$ que avaliou qualidade de vida de 825 estudantes em seis cursos de Enfermagem da Região Sul do Brasil, o maior escore médio foi atribuído ao domínio relações sociais (70), e o menor escore médio para o domínio meio ambiente (55). Considerou-se que a baixa avaliação do domínio meio ambiente poderia estar relacionada à insegurança e à incerteza vividas na contemporaneidade da sociedade brasileira.

A Tabela 3 apresenta os escores médios da qualidade de vida para o total dos acadêmicos de Enfermagem estudados, segundo associações entre características sociodemográficas. Ocorreram associações significativas entre o sexo e os domínios físico e psicológico, com maiores escores médios para os acadêmicos do sexo masculino no domínio físico $(79,9 \pm 9,8)$ e psicológico $(77,6 \pm 6,3)$, em relação ao sexo feminino (domínio físico $=71,0 \pm 13,2$ e domínio psicológico $=67,3 \pm 12,7)$.

O instrumento utilizado para coleta de dados neste estudo contempla, no domínio físico, aspectos relacionados à dor e desconforto, dependência de medicação ou de tratamentos, energia e fadiga, mobilidade, sono e repouso, atividades da vida cotidiana e capacidade de trabalho. Para o domínio psicológico, contempla sentimentos positivos, espiritualidade/religião/ crenças pessoais, pensar, aprender, memória e concentração, imagem corporal e aparência, autoestima e sentimentos negativos ${ }^{16}$. 
Tabela 3 - Escores médios da qualidade de vida do WHOQOL-bref para o total dos acadêmicos de Enfermagem estudados, segundo associações entre características sociodemográficas (Paraná, 2007)

\begin{tabular}{|c|c|c|c|c|c|}
\hline \multirow{2}{*}{ Variáveis } & \multicolumn{5}{|c|}{ Escores médios de qualidade de vida (DP) } \\
\hline & n (\%) & Físico & Psicológico & Relações sociais & Meio ambiente \\
\hline \multicolumn{6}{|l|}{ Sexo } \\
\hline Feminino & $54(80,6)$ & $71,0(13,2)^{*}$ & $67,3(12,7)^{*}$ & $70,1(16,7)$ & $59,8(13,7)$ \\
\hline Masculino & $13(19,4)$ & $79,9(9,8)^{*}$ & $77,6(6,3)^{*}$ & $76,3(9,5)$ & $64,4(6,2)$ \\
\hline \multicolumn{6}{|l|}{ Idade } \\
\hline Até 20 anos & $36(53,7)$ & $71,0(12,1)$ & $69,2(12,2)$ & $69,2(15,0)$ & $61,5(11,5)$ \\
\hline$>20$ anos & $31(46,3)$ & $74,6(12,7)$ & $69,5(12,7)$ & $73,7(16,4)$ & $59,8(14,0)$ \\
\hline \multicolumn{6}{|l|}{ Estado civil } \\
\hline Solteiro(a) & $61(91,0)$ & $72,3(13,4)$ & $68,6(12,5)$ & $70,4(15,6)$ & $60,5(13,1)$ \\
\hline Casado(a) & $6(8,9)$ & $76,8(8,1)$ & $76,4(8,2)$ & $80,6(15,5)$ & $62,5(6,6)$ \\
\hline \multicolumn{6}{|c|}{ Renda per capita média familiar ${ }^{\dagger}$} \\
\hline Até $1 \mathrm{SM}$ & $15(23,4)$ & $70,9(11,4)$ & $70,6(10,0)$ & $69,4(14,3)$ & $57,5(11,4)$ \\
\hline Mais de $1 \mathrm{SM}$ & $49(76,6)$ & $74,2(12,8)$ & $69,8(12,6)$ & $72,3(16,2)$ & $61,6(13,0)$ \\
\hline \multicolumn{6}{|l|}{ Procedência } \\
\hline Cidade local & $26(38,8)$ & $71,6(14,1)$ & $67,6(13,0)$ & $66,7(15,8)$ & $59,5(13,7)$ \\
\hline Outra cidade & $41(61,2)$ & $73,4(12,4)$ & $70,4(11,9)$ & $74,2(15,1)$ & $61,4(12,1)$ \\
\hline \multicolumn{6}{|l|}{ Com quem reside } \\
\hline Sozinho(a) & $9(13,4)$ & $77,8(15,4)$ & $71,8(14,1)$ & $74,1(12,1)$ & $64,9(11,8)$ \\
\hline Familiares/outros & $58(86,6)$ & $71,9(12,6)$ & $69,0(12,1)$ & $70,8(16,2)$ & $60,0(12,8)$ \\
\hline
\end{tabular}

$\mathrm{DP}=$ desvio padrão; $\mathrm{SM}=$ salário mínimo.

* Significativo para $\mathrm{p}<0,05$, teste $t$ de Student.

${ }^{\dagger}$ Trềs dos respondentes não informaram.

Sobre aspectos relacionados a relatos de doenças, em estudo sobre qualidade de vida, $39 \%$ dos acadêmicos de Enfermagem pesquisados relataram ter algum tipo de doença, principalmente problema nervoso crônico ou emocional, depressão e doença de pele ${ }^{2}$.

Mensurando os sintomas de depressão mais freqüentes entre 99 discentes de Enfermagem do primeiro ao quarto ano, $41,41 \%$ apresentaram grau de depressão variando de leve até grave. Os sintomas mais freqüentes foram auto-acusação, irritabilidade e fadiga. A pesquisa buscou articular a questão de gênero, presente na Enfermagem enquanto profissão, e a depressão enquanto um transtorno que acomete com maior freqüência a mulher, principalmente aquela que trabalha em jornada integral. Com relação aos alunos do curso de graduação em Enfermagem, predominantemente mulheres, a grade curricular faz com que eles permaneçam em torno de 9 horas diárias em atividades de ensino, situação semelhante à jornada integral de trabalho ${ }^{26}$.

Telles Filho et al. ${ }^{25}$, estudando estresse em discentes de Enfermagem, relatou que as expectativas mais citadas para o futuro, entre alunos do último período da graduação desse curso, foram referentes a emprego, trabalho, remuneração salarial, vínculo empregatício urgente e realização profissional. Verificando as características evidenciáveis de estresse nesses discentes, observou-se no nível sempre, 39,1\% dos indivíduos responderam sentir desgaste ao final do dia, $34,8 \%$ apresentavam pensamentos que provocavam ansiedade e $30,4 \%$ apresentavam esgotamento emocional.

As diferenças observadas nos resultados deste estudo, com escores médios de qualidade de vida menores para as mulheres nos domínios físico e psicológico, e o fato de a área da saúde ser relativamente desgastante por lidar com vidas em sofrimento, apontam para a necessidade de maior atenção voltada ao processo de formação dos acadêmicos de Enfermagem, especialmente as mulheres.

Como, no contexto da Enfermagem, o processo do cuidar pode causar ansiedade, seria importante favorecer o processo de autoconhecimento e apoio aos estudantes sobre questões como medos e ansiedades, próprios do cuidar de si e dos outros ${ }^{6}$.

De fato, conforme relato de Esperidião e Munari ${ }^{3}$, "o processo de formação acadêmica não tem 
privilegiado aspectos que possibilitem o fortalecimento emocional dos futuros profissionais de saúde, num nítido movimento de desconsideração dos agravos produzidos pelas situações ansiogênicas as quais os alunos estão continuamente expostos".

O processo de cuidar coloca tanto professor como aluno em contato direto com pessoas em sofrimento. Por isso, ressalta-se a importância do papel do professor no aconselhamento e escuta do aluno, para que este possa trabalhar transferência e contra-referência, e elaborar vivências no processo de cuidar de si e dos outros ${ }^{6}$.

A Tabela 4 apresenta as associações entre as variáveis sociodemográficas dos acadêmicos do primeiro e quarto anos. Observam-se diferenças para idade e renda, com o primeiro ano apresentando alunos mais jovens (com até 20 anos) e com menor renda média familiar mensal (até um salário mínimo) em relação ao quarto ano.

Tabela 4 - Distribuição dos acadêmicos de Enfermagem por ano do curso (primeiro e quarto), segundo associações entre variáveis sociodemográficas (Paraná, 2007)

\begin{tabular}{|c|c|c|c|c|c|}
\hline \multirow[t]{2}{*}{ Variáveis } & \multicolumn{2}{|c|}{$\begin{array}{c}\text { Primeiro ano } \\
(n=34)\end{array}$} & \multicolumn{2}{|c|}{$\begin{array}{l}\text { Quarto ano } \\
(n=33)\end{array}$} & \multirow[t]{2}{*}{$\mathbf{p}$} \\
\hline & $\mathbf{n}$ & $(\%)$ & $\mathbf{n}$ & $(\%)$ & \\
\hline \multicolumn{6}{|l|}{ Sexo } \\
\hline Feminino & 26 & $(48,1)$ & 28 & $(51,9)$ & \\
\hline Masculino & 8 & $(61,5)$ & 5 & $(38,5)$ & 0,54 \\
\hline \multicolumn{6}{|l|}{ Idade } \\
\hline Até 20 anos & 26 & $(72,2)$ & 10 & $(27,8)$ & \\
\hline$>20$ anos & 8 & $(25,8)$ & 23 & $(74,2)$ & 0,00 \\
\hline \multicolumn{6}{|l|}{ Estado civil } \\
\hline Solteiro(a) & 31 & $(50,8)$ & 30 & $(49,2)$ & \\
\hline Casado(a) & 3 & $(50,0)$ & 3 & $(50,0)$ & 1 \\
\hline \multicolumn{6}{|c|}{ Renda per capita média familiar* } \\
\hline Até $1 \mathrm{SM}$ & 12 & $(80,0)$ & 3 & $(20,0)$ & \\
\hline$>1 \mathrm{SM}$ & 22 & $(44,9)$ & 27 & $(55,1)$ & 0,02 \\
\hline \multicolumn{6}{|l|}{ Procedência } \\
\hline Cidade local & 13 & $(50,0)$ & 13 & $(50,0)$ & \\
\hline Outra cidade & 21 & $(51,2)$ & 20 & $(48,6)$ & 1 \\
\hline \multicolumn{6}{|l|}{ Com quem reside } \\
\hline Sozinho & 4 & $(44,4)$ & 5 & $(55,6)$ & \\
\hline Familiares/outro & 30 & $(51,7)$ & 28 & $(48,3)$ & 0,73 \\
\hline
\end{tabular}

$\mathrm{SM}=$ salário mínimo.

Significativo para $\mathrm{p}<0,05$, teste exato de Fisher.

* Três respondentes do quarto ano não informaram.

$\mathrm{O}$ ingresso na universidade, para a maioria dos jovens, ocorre em uma fase de transição da adolescência para a vida adulta, em geral com muitas dúvidas e incertezas. $\mathrm{O}$ ambiente escolar, por constituir uma mudança nos estilos de vida, exige também dos alunos um período de adaptação ${ }^{29}$. Geralmente chegam ao meio acadêmico sob forte estresse e pressão, em função do vestibular, com uma série de expectativas e desejos, próprios do momento de suas vidas. Contudo, deparam-se muitas vezes com uma realidade nem sempre esperada, em relação ao curso e às condições de ensino, percebendo-se em uma nova etapa de suas vidas, onde são chamados à responsabilidade 3 .

A Tabela 5 apresenta a comparação dos escores médios dos domínios da qualidade de vida entre os acadêmicos do primeiro e quarto anos, sendo que não houve diferença significativa.

A diferença significativa observada em idade e renda média entre os dois grupos de acadêmicos poderia influenciar nas avaliações dos domínios da qualidade de vida, o que não foi observado neste estudo. Esse achado reforça o entendimento de 
Tabela 5 - Escores médios dos domínios da qualidade de vida do WHOQOL-bref para acadêmicos de Enfermagem do primeiro e quarto anos. Paraná, 2007

\begin{tabular}{lccccc}
\hline \multirow{2}{*}{ Domínios } & \multicolumn{2}{c}{$\begin{array}{c}\text { Primeiro ano } \\
(\mathbf{n}=\mathbf{3 4})\end{array}$} & \multicolumn{2}{c}{$\begin{array}{c}\text { Quarto ano } \\
(\mathbf{n}=\mathbf{3 3})\end{array}$} & $\mathbf{p}$ \\
\cline { 2 - 5 } & Escore médio & $(\mathbf{D P})$ & Escore médio & $(\mathbf{D P})$ & \\
\hline Físico & 71,85 & $(13,15)$ & 73,60 & $(13,09)$ & 0,59 \\
Psicológico & 69,49 & $(12,30)$ & 69,19 & $(12,58)$ & 0,92 \\
Relações sociais & 72,06 & $(16,52)$ & 70,45 & $(15,03)$ & 0,68 \\
Meio ambiente & 60,40 & $(11,46)$ & 61,00 & $(13,97)$ & 0,85 \\
\hline
\end{tabular}

$\mathrm{DP}=$ desvio padrão.

Significativo para $\mathrm{p}<0,05$, teste $t$ de Student.

qualidade de vida como subjetiva e pessoal, com a valorização da opinião do indivíduo9.

Em estudo sobre percepção de qualidade de vida realizado com estudantes da graduação em Enfermagem, com abordagem qualitativa, observouse que as principais situações promotoras de qualidade de vida foram as experiências extracurriculares, a relação professor-aluno e o relacionamento entre alunos. Já as não promotoras de qualidade de vida foram a falta de acolhimento por parte dos professores e enfermeiros de campo, a falta de integração com equipe e alunos de outros cursos e a carga horária excessiva para o aluno trabalhador ${ }^{6}$.

Ainda conforme dados da Tabela 5, os maiores escores médios foram para o domínio relações sociais $(72,06 \pm 16,52)$ para os acadêmicos do primeiro ano, e para o domínio físico $(73,60 \pm 13,09)$ para os acadêmicos do quarto ano. Os menores escores médios avaliados foram para o domínio meio ambiente em ambos os grupos, com escore médio de 60,40 $(\mathrm{DP} \pm 11,46)$ para o primeiro ano e $61(\mathrm{DP} \pm 13,97)$ para o quarto ano.

Beuter et al..$^{5}$ pesquisaram o lazer na vida dos acadêmicos de graduação em Enfermagem, já que, além das atividades acadêmicas, a utilização do tempo livre pode influenciar escolhas futuras do estudante. Assim, os acadêmicos de Enfermagem, entendendo e valorizando o lazer, em uma dimensão mais abrangente, nos diversos momentos de suas vidas, como um cuidado de si, podem trazer conforto, bemestar, alívio, alegria e tranqüilidade, oportunizando o cuidado humanizado do outro.

Os baixos escores médios do domínio meio ambiente para os acadêmicos pesquisados neste estudo trazem preocupação, já que o ritmo do capitalismo impõe um cotidiano agitado aos indivíduos, com valorização apenas do futuro. E um mercado de trabalho cada vez mais exigente faz com que o tempo seja dedicado exclusivamente às atividades do futuro profissional, o que pode levar a um distanciamento das coisas que lhe dão prazer 5 .

Tanto o cenário econômico do país, com falta de perspectivas de trabalho, como a vivência dos alunos nos campos de estágio, em um sistema de saúde pouco voltado ao atendimento das reais necessidades da população, não são fatores estimulantes para um desempenho otimista e não favorecem a auto-estima dos jovens que estão prestes a entrar no mercado de trabalho' ${ }^{24}$.

Além disso, aspectos relacionados ao ambiente físico precisam ser considerados. Em pesquisa realizada com 2.613 respondentes, entre professores, funcionários e alunos, foram avaliados aspectos do ambiente físico do Campus da Universidade de Brasília. Na avaliação global, foram avaliados como ruim a reciclagem de papel, vidro e plásticos, iluminação externa, e proteção contra furtos. As prioridades para melhorias citadas foram aspectos de segurança, tais como iluminação externa, proteção contra furtos, segurança pessoal, além de transporte coletivo, segurança de trânsito e qualidade da água ${ }^{30}$.

\section{Conclusões}

O total de acadêmicos pesquisados foi constituído predominantemente por mulheres, jovens, solteiras, com renda per capita média familiar de mais de um salário mínimo, procedentes de outras cidades e que residiam com a família ou em república/pensionato. $\mathrm{O}$ maior escore médio de qualidade de vida foi para o domínio físico e o menor, para o domínio meio ambiente. $\mathrm{Na}$ correlação com variáveis sociodemográficas, apenas o sexo apresentou correlação com a qualidade de vida, com maiores escores médios para os acadêmicos do sexo masculino nos domínios físico e psicológico. 
$\mathrm{Na}$ análise comparativa entre os acadêmicos do primeiro e quarto anos, apesar das diferenças significativas para idade e renda, com o primeiro ano apresentando alunos mais jovens e com menor renda média familiar mensal em relação ao quarto ano, não houve diferença significativa nos domínios da qualidade de vida.

Os resultados deste estudo sugerem que os acadêmicos de Enfermagem merecem atenção quanto aos aspectos da qualidade de vida, principalmente as mulheres nos aspectos físico e psicológico.

A Enfermagem assume um papel muito importante tanto na manutenção como na promoção da saúde. Além disso, coloca em prática o pensar pelas ações do cuidar e ensinar, por ser uma profissão que possui singularidades nos seus conhecimentos ${ }^{31}$.

Dessa forma, a formação desses profissionais voltada apenas para o desempenho técnico específico não pode mais ser aceita pelas instituições de ensino superior, devendo voltar-se para a formação do profissional cidadão, com competência técnica e científica, e, sobretudo, com ampla visão da dimensão humana ${ }^{32}$.

Deve-se atentar para o ensino centrado na pessoa, considerando a competência técnica e racional do aluno, mas também sua totalidade, entendendo-o como alguém que possui atributos pessoais e que experimenta diversos sentimentos durante suas ações profissionais ${ }^{3}$.

Também não se deve esquecer o valor do docente na vida do acadêmico, tanto para o desempenho profissional quanto para o pessoal. Afinal, o docente acompanha o acadêmico em grande parte do tempo, desde sua entrada na universidade até sua saída, para que aspectos negativos de sua qualidade de vida vividos ou ocorridos durante a formação não interfiram de modo a prejudicar sua carreira profissional.

Espera-se que este estudo sirva de motivação para outras pesquisas de avaliação de qualidade de vida de acadêmicos de Enfermagem, com comparação entre grupos e utilização de diferentes abordagens metodológicas. Esses resultados podem servir como fontes de informação para elaboração de estratégias de suporte e enfrentamento para os acadêmicos, bem como para implantação e manutenção de espaços formalizados dedicados às suas reais necessidades, gratuitos, de fácil acesso e que respeitem os aspectos éticos.

\section{Referências}

1. Backes VMS, Nietsche EA, Camponogara S, Fraga RS, Cerezer RC. A educação continuada dos alunos egressos: compromisso da universidade? Rev Bras Enferm. 2002;55(2):200-4.
2. Saupe R, Nietche EA, Cestari ME, Giorgi MD, Krahl M. Qualidade de vida dos acadêmicos de enfermagem. Rev Latino-Am Enfermagem. 2004;12(4):636-42.

3. Esperidião E, Munari DB. Holismo só na teoria: a trama de sentimentos do acadêmico de enfermagem sobre sua formação. Rev Esc Enferm USP. 2004;38(3):332-40.

4. Jorge MSB, Rodrigues ARF. Serviços de apoio ao estudante oferecidos pelas escolas de enfermagem no Brasil. Rev Latino-Am Enfermagem. 1995;3(2):59-68.

5. Beuter M, Alvim NAT, Mostardeiro SCTS. O lazer na vida de acadêmicos de enfermagem no contexto do cuidado de si para o cuidado do outro. Texto \& Contexto Enferm. 2005;14(2):222-8.

6. Oliveira RA, Ciampone MHT. A universidade como espaço promotor de qualidade de vida: vivências e expressões dos alunos de enfermagem. Texto \& Contexto Enferm. 2006;15(2):254-61.

7. Farquhar M. Definitions of quality of life: a taxonomy. J Adv Nurs. 1995;22(3):502-8.

8. Meeberg GA. Quality of life: a concept analysis. J Adv Nurs. 1993;18(1):32-8.

9. Paschoal SMP. Qualidade de vida no idoso: elaboração de um instrumento que privilegia sua opinião [dissertação]. São Paulo: Faculdade de Saúde Pública; 2001.

10. Seidl EMF, Zannon CMLC. Qualidade de vida e saúde: aspectos conceituais e metodológicos. Cad Saude Publica. 2004;20(2):5808 .

11. Carr AJ, Gibson B, Robinson PG. Is quality of life determined by expectations or experience? BMJ. 2001;322(7296):1240-3.

12. Holmes S, Dickerson J. The quality of life: design and evaluation of a self-assessment instrument for use with cancer patients. Int J Nurs Stud. 1987;24(1):15-24

13. World Health Organization. WHOQOL: measuring quality of life. Genebra: WHO; 1997. MAS/MNH/PSF/97.4.

14. Pereira MG. Epidemiologia: teoria e prática. Rio de Janeiro: Guanabara Koogan; 1995.

15. Brasil, Ministério da Saúde. Conselho Nacional de Saúde, Comissão Nacional de Ética em Pesquisa (CONEP). Normas para Pesquisa envolvendo seres humanos/Res. CNS 196/96 e outras). Brasília: Ministério da Saúde; 2000. http://conselho.saude.gov.br/comissao/ conep/resolucao.html. Acessado em 26 nov 2008.

16. Fleck MPA, Louzada S, Xavier M, Chachamovich E, Vieira G, Santos L, et al. Aplicação da versão em português do instrumento abreviado de avaliação de qualidade de vida "WHOQOL - bref". Rev Saude Publica. 2000;34(2):178-83.

17. Fleck MPA, Fachel O, Louzada S, Xavier M, Chachamovich E, Vieira $\mathrm{G}$, et al. Desenvolvimento da versão em português do instrumento de avaliação de qualidade de vida da OMS (WHOQOL100). Rev Bras Psiquiatr. 1999;21(1):19-28.

18. Guyatt GH, Feeny DH, Patrick DL. Measuring health-related quality of life. Ann Intern Med. 1993;118(8):622-9.

19. Testa MA, Simonson DC. Assessment of quality of life outcomes. N Engl J Med. 1996;334(13):835-40.

20. Berlim MT, Fleck MP. "Quality of life": a brand new concept for research and practice in psychiatry. Rev Bras Psiquiatr. 2003;25(4):249-52.

21. Wetterich NC, da Costa Melo MR. Sociodemographic profile of undergraduate nursing students. Rev Lat Am Enfermagem. 2007; 15(3):404-10.

22. Kawakame PMG, Miyadahira MKK. Qualidade de vida de estudantes de graduação em enfermagem. Rev Esc Enferm USP. 2005;39(2):164-72.

23. Trovo MM, Silva Leão MJP, Eliseth Ribeiro E. Terapias alternativas/complementares no ensino público e privado: análise do conhecimento dos acadêmicos de enfermagem. Rev Latino-Am Enfermagem. 2003;11(4):483-9.

24. Furegato ARF, Silva EC, Campos MC, Cassiano RPT. Depressão e auto-estima entre os acadêmicos de Enfermagem. Rev Psiquiatria Clin. 2006;33(5):239-44.

25. Telles Filho PCP, Pires E, Araújo GA. Características evidenciáveis de estresse em discentes de Enfermagem. Rev Latino-Am Enfermagem. 1999;7(2):91-3. 
26. Santos TM, Almeida AO, Martins HO, Moreno V. Aplicação de um instrumento de avaliação do grau de depressão em universitários do interior paulista durante a graduação em enfermagem. Acta Scient. 2003;25(2):171-6

27. Santos CE, Leite MMJ. O perfil do aluno ingressante em uma universidade particular da cidade de São Paulo. Rev Bras Enferm. 2006;59(2):154-6.

28. Teixeira E, Vale EG, Fernandes JD, De Sordi MRL. Trajetória e tendências dos cursos de enfermagem no Brasil. Rev Bras Enferm. 2006;59(4):479-87.
29. Scherer ZAP, Scherer EA, Carvalho AMP. Reflexões sobre o ensino da enfermagem e os primeiros contatos do aluno com a profissão. Rev Latino-Am Emfermagem. 2006;14(2):285-91.

30. Günther H, Puente KE. Ambiente físico e qualidade de vida no campos da UnB. http://www.unb.br/ip/lpa/pdf/tlp19940307.pdf . Acessado dez 2008.

31. Camacho ACLF, Espírito Santo FH. Refletindo sobre o cuidar e o ensinar na enfermagem. Rev Latino-Am Enfermagem. 2001;9(1):137.

32. Camillo SO, Silva AL, Nascimento AJ. Percepções do Graduando de Enfermagem sobre a dimensão humana no seu aprendizado. Rev Latino-Am Enfermagem. 2007;15(2):207-13. 